\title{
ARTICLE
}

\section{Towards uniformly oriented diatom frustule monolayers: Experimental and theoretical analyses}

\author{
Aobo $\mathrm{Li}^{1}$, Wenqiang Zhang ${ }^{1, *}$, Reza Ghaffarivardavagh ${ }^{1}$, Xiaoning Wang ${ }^{1}$, Stephan W. Anderson ${ }^{2}$ and Xin Zhang $^{1}$
}

Diatoms are unicellular, photosynthetic algae that are ubiquitous in aquatic environments. Their unique, three-dimensional (3D) structured silica exoskeletons, also known as frustules, have drawn attention from a variety of research fields due to their extraordinary mechanical properties, enormous surface area, and unique optical properties. Despite their promising use in a range of applications, without methods to uniformly control the frustules' alignment/orientation, their full potential in technology development cannot be realized. In this paper, we realized and subsequently modeled a simple bubbling method for achieving large-area, uniformly oriented Coscinodiscus species diatom frustules. With the aid of bubble-induced agitations, close-packed frustule monolayers were achieved on the water-air interface with up to nearly $90 \%$ of frustules achieving uniform orientation. The interactions between bubble-induced agitations were modeled and analyzed, demonstrating frustule submersion and an adjustment of the orientation during the subsequent rise towards the water's surface to be fundamental to the experimentally observed uniformity. The method described in this study holds great potential for frustules' engineering applications in a variety of technologies, from sensors to energy-harvesting devices.

Keywords: diatom; manipulation; monolayer; uniform

Microsystems \& Nanoengineering (2016) 2, 16064; doi:10.1038/micronano.2016.64; Published online: 5 December 2016

\section{INTRODUCTION}

It has been several decades since diatoms first drew the attention of researchers ${ }^{1}$. Diatoms are unicellular, photosynthetic algae notable for the remarkable exoskeletons enclosing their cytoplasm ${ }^{2,3}$. These exoskeletons, also known as frustules, are arranged in intricate hierarchical pore structures formed from amorphous biosilica. In some species, such as Coscinodiscus species (C. $s p$.) diatoms, the exoskeleton surfaces include up to three layers of pore structures, with pore sizes ranging from $60 \mathrm{~nm}$ to $1 \mu \mathrm{m}$. These hierarchical, porous structures give rise to the diatom frustules' extraordinary mechanical strength ${ }^{4}$, enormous surface area ${ }^{5}$, and unique optical properties ${ }^{6,7}$.

The development of the use of diatoms in a variety of technological applications is ongoing and, although abundant sizes and shapes of frustules are available, circular microscopic diatoms (such as C. sp.) are especially popular due to their complex hierarchical porous system and centrosymmetric structures ${ }^{8,9}$. To date, the use of diatoms as key components in a variety of application areas has been reported. For example, in applications to sensing technology, diatoms have been employed as the sensing components in immunoassays and gas sensors ${ }^{10-14}$. Furthermore, in work by Lin et $a l^{11}{ }^{11}$, diatom frustules have been employed in electrochemical impedance sensing and have been found to significantly improve performance, including sensitivity, response time, and dynamic range, compared with existing approaches. In optical research, it has been demonstrated that diatom frustules behave as optical crystals, focusing light due to their micron and sub-micron scale pore arrays ${ }^{15,16}$. In addition, it has been reported that frustules have the unique ability to trap light due to their hierarchical pore structures ${ }^{7,17,18}$, a property that has been successfully leveraged in enhancing dye-sensitized solar cell performance ${ }^{17}$.

Given the 3D shape of diatom frustules, such as the commonly employed C. sp. frustules, the alignment and orientation of these biological materials is fundamental to optimizing their utility in a variety of applications. For example, in the application to biosensors, in which diatom frustules have been employed as bonding sites ${ }^{19}$, the ability to uniformly orient diatom frustules (especially in a 'concave side facing down' manner) may increase the utilization of the micro-nanostructures, thus increasing the bonding area. Moreover, in optical applications ${ }^{7}$, uniformly oriented frustules have the potential to form light focusing/ trapping layers, thereby allowing more than just single diatoms to act as optical components, increasing the potential scale and efficiency of these devices. Importantly, the ability to manipulate the frustules on a large scale, combined with simple arraying techniques ${ }^{20}$, may enable the development of practical, real-world applications using diatoms. However, despite the promise of the use of diatom frustules in a variety of areas, the unique properties of diatoms have typically only been studied on the scale of a single diatom frustule (requiring only individual diatom alignment) $^{6-8,10-16}$ or as bulk materials (requiring no significant alignment) ${ }^{17}$.

To arrange the diatom frustules into large-area monolayers, the use of self-assembly techniques may be considered. A variety of self-assembly approaches have been reported, including the assembly of particles with a range of sizes and shapes ${ }^{21-23}$. Special attention has been paid to the 'floating assembly' of

\footnotetext{
${ }^{1}$ Department of Mechanical Engineering, Boston University, Boston, MA 02215, USA and ${ }^{2}$ Department of Radiology, Boston University Medical Center, Boston, MA 02118, USA. Correspondence: Stephan W. Anderson (Stephan.Anderson@bmc.org) or Xin Zhang (xinz@bu.edu)

*Present address: College of Engineering, China Agricultural University, Beijing 100083, China.

Received: 1 December 2015; revised: 16 July 2016; accepted: 31 July 2016
} 


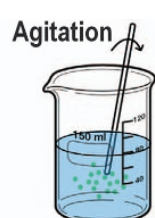

A. Frustule hydrophobic treatment in NXT-110 mold release agent

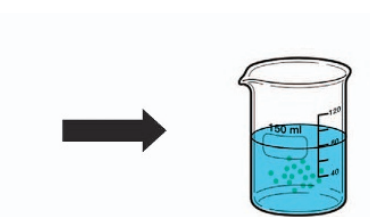

B. Surface modified frustules cleaned and dispersed with ethanol

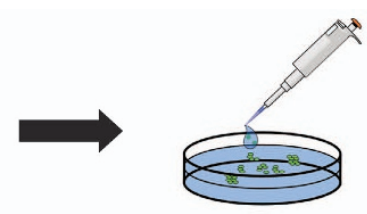

C. Frustules dropped to water to form frustule monolayer
D1. $\mathrm{N}_{2}$ bubbles pumped under water for orientation adjustments
D2. $\mathrm{N}_{2}$ bubbles pumped under water with filter paper covering the petri dish
D3. DI water dropped to floating

frustules for their submersion

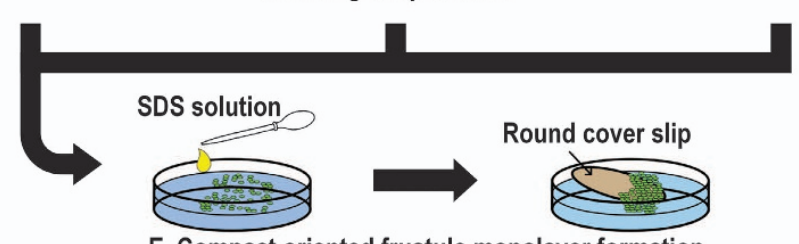

E. Compact oriented frustule monolayer formation

Figure 1 Process of uniformly arranging diatoms into a concave-down configuration. Three different approaches were employed to investigate the resultant concave-down frustule ratios (D1, D2, and D3).

micronanoparticles floating on liquid-air interfaces ${ }^{24-26}$, as this technique is technically relatively simple and produces high-quality monolayers or even 3D assemblies of particles. It has been reported that the success of the floating assembly results from the capillary forces between the floating particles ${ }^{27,28}$. Although initial progress has been reported in achieving the capacity for 'batch control' of frustule orientation as well as compactness as they form frustule monolayers on a flat substrate surface ${ }^{29,30}$, little attention has been paid to achieving uniform orientation in synchrony in large-area diatom monolayers. Herein, a universal method to obtain large-area, uniformly oriented diatom frustule monolayers is developed, with simulations and theoretical analysis conducted to illuminate the underlying principles.

\section{MATERIALS AND METHODS}

\section{Diatom frustule preparation}

Diatom frustules (C. sp.; catalog No. CCMP 1583, Bigelow Laboratory for Ocean Sciences, East Boothbay, ME, USA) were collected from $20 \mathrm{I}$ of bulk culturing medium. The diatoms were initially settled and concentrated to a volume of $15 \mathrm{ml}$ and subsequently cleaned with sulfuric acid. For this purpose, $350 \mathrm{ml}$ of concentrated sulfuric acid was added to the condensed diatom culture medium; the mixture was then heated to $60^{\circ} \mathrm{C}$ for $40 \mathrm{~min}$. After cooling, the frustules were settled, and the supernatant of the solution was removed. The mixture was then added to deionized (DI) water for dilution and further settling. This dilutionsettling procedure was repeated 4 to 5 times for further purification and concentration ${ }^{31}$ before the $\mathrm{pH}$ value of the solution reached neutral, after which the diatoms were stored in DI water. For subsequent imaging, the diatoms were first dried and dispensed onto clean silicon wafers, sputter coated with an $\sim 10 \mathrm{~nm}$ gold/palladium alloy layer (Cressington 108 sputter coater, Cressington Scientific Instruments, Watford, UK) and then examined using a scanning electron microscope (Zeiss Supra 55VP, Zeiss, Thornwood, NY, USA).

\section{Uniformly oriented diatom monolayer formation}

To form compact, self-assembled monolayers of frustules on a water surface, the frustule surface was first rendered hydrophobic. For this purpose, $3 \mathrm{ml}$ of NXT-110 mold release agent (Nanonex Corporation, Monmouth Junction, NJ, USA) were employed; after two parts of the agent (NXT-110 A \& B, volume ratio $A: B=1: 2$ ) were mixed, $0.1 \mathrm{~g}$ dry diatom frustules were immediately added to the solution. After $1 \mathrm{~min}$ of agitation, the frustules were filtered and rinsed with ethanol (Figure 1, A and B). The modified frustules were then dispersed in ethanol (weight ratio frustules: ethanol $=1: 10$ ).

Hydrophilic thin glass cover slips (diameter $\sim 13 \mathrm{~mm}$ ) were prepared for the experiments involving the collection of the frustule monolayers by first soaking in $10 \%$ sodium dodecyl sulfate-water solution (SDS) for $2 \mathrm{~h}$.

The prepared frustule suspension was slowly added, drop-bydrop, to DI water in a Petri dish with a diameter of $30 \mathrm{~mm}$ (Figure 1, C). The frustules floated on the water's surface and formed a frustule layer. To adjust the frustule orientation, $\mathrm{N}_{2}$ bubbles were blown from $\sim 3 \mathrm{~mm}$ beneath the water's surface for 1 min for each group (Figure 1, D1). The $\mathrm{N}_{2}$ gas source was maintained at $5 \mathrm{psi}$ and $0.1 \mathrm{SCFH}$ throughout. The inner diameter of the $\mathrm{N}_{2}$ source nozzles was varied $(0.076,0.26,0.52,0.66$, and $1 \mathrm{~mm}$ ) to achieve different bubble sizes (see Results section). Statistics regarding the bubble sizes were obtained from slowmotion videos of the bubbling process. The bubble source was moved throughout the water-filled Petri dish to ensure agitation of the entire surface of the water by the $\mathrm{N}_{2}$ bubbles. To form a compact frustule monolayer, $\sim 20 \mu \mathrm{l}$ of $2 \%$ SDS solution was added to the water, and the frustules were compacted, forming a 
a

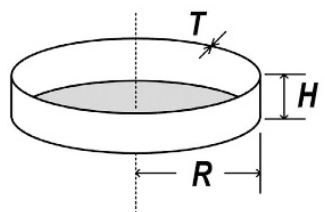

C

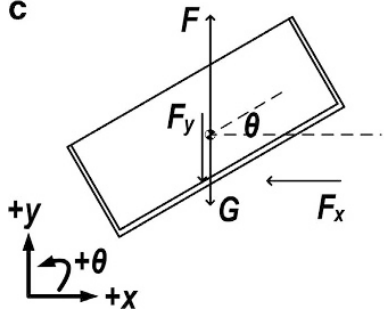

b
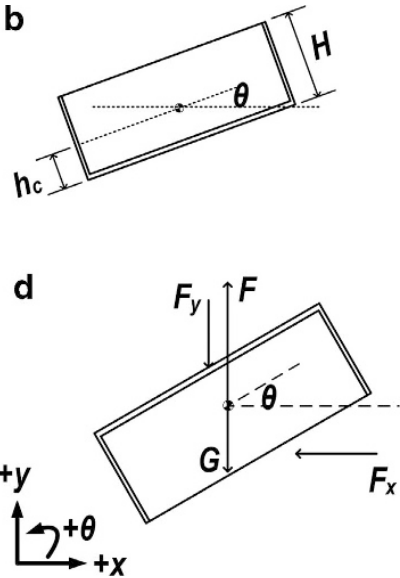

Figure 2 Theoretical analysis of diatom frustule interaction with water as it rises towards the surface following submersion. (a) An analytical model of a single diatom frustule. (b) A cross-section of the frustule model: $h_{c}$ denotes the distance between the mass center and convex surface of a frustule. (c) Forces exerted on a rising 'concave-up' oriented diatom frustule: $F$ denotes the buoyance force, $G$ the gravitational force, $F_{x}$ and $F_{y}$ the drag forces resulting from the corresponding relative water flow. (d) Forces exerted on a rising 'concave-down' diatom frustule.

monolayer, which could then be lifted from the water's surface using a modified cover slip (Figure 1, E). In the control groups, the floating diatoms were not agitated by the bubbles.

During this bubbling process, water jets from the rupturing bubbles were observed. Therefore, corresponding experiments were conducted to examine the effect of the water jets on the diatom frustule orientation. In these experiments, in addition to the previously described bubbling set-up, filter paper was used to cover the petri dish as $\mathrm{N}_{2}$ gas was pumped in (Figure 1, D2). In addition, in a control group, the experiments were performed without the filter paper. For these experiments, a nozzle diameter of $0.66 \mathrm{~mm}$ was used throughout.

In addition to the approach using $\mathrm{N}_{2}$ gas bubbles, an alternative agitation method was employed to further evaluate the interaction between water and diatoms. In this alternative method, the diatom frustules were initially dispersed along the water's surface, forming diatom monolayers as described above. Subsequently, water droplets were released from $3 \mathrm{~mm}$ above the water's surface, thereby impacting the floating diatoms. During this step, $2 \mathrm{ml}$ pipettes with nozzle inner diameters of $\sim 1.6 \mathrm{~mm}$ were employed, and water droplets with diameters of $\sim 4 \mathrm{~mm}$ were generated. For each group of experiments, a total of $\sim 2 \mathrm{ml}$ of water was used to impact the floating diatoms along the water's surface (Figure 1, D3). Following this alternative method of agitation, the frustules were collected using the SDS solution as described above for subsequent observation.

For each method described above, at least 4 groups of experiments were performed. The specimens were subsequently observed, and the relative numbers of frustules with the concave side facing upwards (concave-up) and downwards (concavedown) were counted using a scanning electron microscope, yielding the statistical data presented in the Results section.

\section{Analytical method for investigating the diatom-orienting phenomenon}

Through careful observation of the bubble agitation procedure, we hypothesized that the disturbance of the water's surface may have resulted in submersion of the frustules in water, followed by a floating (rising) of the diatoms back towards the water's surface. Hence, using an analytical approach, we studied how submerged diatoms interacted with the surrounding water as they floated towards the water surface, focusing on the orientation of the diatoms during this procedure.

To this end, we developed a mechanical model of a submerged diatom frustule (Figure 2) with different initial conditions of depth and angular deviation ( $\theta$ in Figure 2 ). We sought to use mechanics approaches to analyze a simplified model of a frustule and provide a qualitative description of the propagation of the frustule's horizontal movement $(x(t))$, vertical movement $(y(t))$, and angular deviation $(\theta(t))$. An increasingly accurate quantitative description of the frustule's movement may also be derived using numerical methods, which will also be discussed in this paper.

For theoretical analysis and the subsequent simulations, a reduced 2D model of the diatom frustule was built (Figures $2 \mathrm{a}$ and b, Supplementary Materials, Supplementary Figure S1). The known conditions of the model and the assumptions are stated as follows. According to our previous observations of the C. sp. diatom frustules, the radius $(R)$, height $(H)$, and thickness $(T)$ of a C. $s p$. diatom frustule were set to $61,17.15$, and $1 \mu \mathrm{m}$, respectively. The frustules' effective density approximates $120-250 \mathrm{~kg} \mathrm{~m}^{-3}$ (Ref. 32); for the computation, we employed a density of $150 \mathrm{~kg} \mathrm{~m}^{-3}$. This geometric model was employed to estimate its related parameters, including mass $(m)$, position of mass center $\left(h_{c}\right.$; Figure $\left.2 \mathrm{~b}\right)$, and moment of inertia $(l)$. To avoid the zerothickness effect of a 2D model, we also introduced a virtual out-ofcross-section-plane 'depth' of the frustule ( $d$; Supplementary Materials, Supplementary Figure S2).

A frustule in water experiences gravitational force $(G$, as shown in Figure 2), buoyancy force $(F)$, and drag forces from the interaction between the diatom and its surrounding water $\left(F_{x}\right.$ and $F_{y}$; Figures $2 \mathrm{c}$ and $\mathrm{d}$ ). The drag force is exerted against the direction in which the diatom is moving and is mainly due to the momentum change of the water moving in $x$ and $y$ directions. Due to viscosity and the frustule's acceleration and deceleration in water, added mass $\left(m_{\mathrm{a}}\right)$ and added momentum of inertia $\left(I_{\mathrm{a}}\right)^{33,34}$ are also considered in this analytical model. Note that the movements of water in both the $x$ and $y$ directions against the frustules will generate component forces along the $x$ and $y$ directions.

For a diatom with its concave side initially facing upward, the force balance equations in the $x$ and $y$ directions are as follows:

$$
\begin{aligned}
& \sum F_{x}=-F_{x x}+F_{x y}=\left(m+m_{a}\right) x^{\prime \prime}(t) \\
& \sum F_{y}=F-F_{y y}+F_{y x}-\left(m+m_{a}\right) g=\left(m+m_{a}\right) y^{\prime \prime}(t)
\end{aligned}
$$

where $x^{\prime \prime}(t)$ and $y^{\prime \prime}(t)$ represent the frustule's acceleration along the $x$ and $y$ directions, respectively, and $F_{i j}$ stands for the component force in the $i$ direction resulting from water flow in the $j$ direction. The forces on the left hand side of Equation (1) can be expressed as follows:

$$
\begin{aligned}
F_{x x}= & 2 \rho R d\left[x^{\prime}(t)\right]^{2} \sin ^{3} \theta(t)+\rho H d\left[x^{\prime}(t)\right]^{2} \cos ^{3} \theta(t) \\
F_{x y}= & \rho d[2 R-H \tan \theta(t)]\left[y^{\prime}(t)\right]^{2} \cos ^{2} \theta(t) \sin \theta(t) \\
& -2 \rho H d\left[y^{\prime}(t)\right]^{2} \sin ^{2} \theta(t) \cos \theta(t) \\
F= & \rho V_{d} g \\
F_{y y}= & \rho d[2 R-H \tan \theta(t)]\left[y^{\prime}(t)\right]^{2} \cos ^{3} \theta(t)+2 \rho H d\left[y^{\prime}(t)\right]^{2} \sin ^{3} \theta(t) \\
F_{y x}= & 2 \rho R d\left[x^{\prime}(t)\right]^{2} \sin ^{2} \theta(t) \cos \theta(t)-\rho H d\left[x^{\prime}(t)\right]^{2} \cos ^{2} \theta(t) \sin \theta(t)
\end{aligned}
$$

where $\rho$ is the density of water, $x^{\prime}(t)$ and $y^{\prime}(t)$ are the frustule velocities in the $x$ and $y$ directions, respectively, $V_{d}$ is the volume of the diatom frustule, and $g$ is the gravitational acceleration. The initial tilt angle $(\theta(0))$ of the diatom frustule with respect to the horizontal plane can be set to varying values, and $\theta(0)=0-90^{\circ}$ was defined as corresponding to the case in which the diatom begins rising with its concave side facing upwards. 
To develop a rotational model of the diatom, we assume that the drag forces are uniformly distributed along the surface of the frustule (Supplementary Material, Supplementary Figure S1). Note that there is a small volume within the frustule (marked as green in Supplementary Figure S1) that is not under the influence of this force, as it is shadowed by the sidewall of the frustule. By summing the torque from all parts of the frustule with respect to the mass center, we derive the total torque and the rotational movement model of the frustule as follows:

$$
\sum M=M_{x}+M_{y}=\left(I+I_{a}\right) \theta^{\prime \prime}(t)
$$

where $M_{x}$ and $M_{y}$ are the torques resulting from the drag forces $F_{x}$ and $F_{y}$, and $\theta^{\prime \prime}(t)$ is the angular acceleration of the frustule. The torques can be further expressed as follows:

$$
\begin{aligned}
M_{x}= & \rho H d\left[x^{\prime}(t)\right]^{2} \cos ^{2} \theta(t)\left(\frac{H}{2}-h_{c}\right) \\
M_{y}= & 2 \rho H d\left[y^{\prime}(t)\right]^{2} \sin ^{2} \theta(t)\left(\frac{H}{2}-h_{c}\right) \\
& +\rho d[2 R-H \tan \theta(t)]\left[y^{\prime}(t)\right]^{2} \cos ^{2} \theta(t) \frac{H \tan \theta(t)}{2}
\end{aligned}
$$

Equations (1) and (3) are coupled, and by solving them together, the linear and rotational movements of the frustule can be determined. Equations (1) and (3) are also appropriate in the case of a frustule with its concave side initially facing downward; however, in this case, the component forces and torques are changed as follows:

$$
\begin{aligned}
F_{x x}= & \rho d[2 R-H \cot \theta(t)]\left[x^{\prime}(t)\right]^{2} \sin ^{3} \theta(t)+2 \rho H d\left[x^{\prime}(t)\right]^{2} \cos ^{3} \theta(t) \\
F_{x y}= & 2 \rho R d\left[y^{\prime}(t)\right]^{2} \cos ^{2} \theta(t) \sin \theta(t)-\rho H d\left[y^{\prime}(t)\right]^{2} \sin ^{2} \theta(t) \cos \theta(t) \\
F_{y y}= & 2 \rho R d\left[y^{\prime}(t)\right]^{2} \cos ^{3} \theta(t)+\rho H d\left[y^{\prime}(t)\right]^{2} \sin ^{3} \theta(t) \\
F_{y x}= & \rho d[2 R-H \cot \theta(t)]\left[x^{\prime}(t)\right]^{2} \sin ^{2} \theta(t) \cos \theta(t) \\
& -2 \rho H d\left[x^{\prime}(t)\right]^{2} \cos ^{2} \theta(t) \sin \theta(t) \\
M_{x}= & -2 \rho H d\left[x^{\prime}(t)\right]^{2} \cos ^{2} \theta(t)\left(\frac{H}{2}-h_{c}\right) \\
& -\rho d\left[2 R-H \cot ^{2} \theta(t)\right]\left[x^{\prime}(t)\right]^{2} \sin ^{2} \theta(t) \frac{H \cot \theta(t)}{2} \\
M_{y}= & -\rho H d\left[y^{\prime}(t)\right]^{2} \sin ^{2} \theta(t)\left(\frac{H}{2}-h_{c}\right)
\end{aligned}
$$

The magnitudes of the added mass and added momentum of inertia were calculated from the reported formula for a rectangular cross-section for in-plane translation and rotation ${ }^{34}$. In this case, $\theta(0)=0-90^{\circ}$ was defined as corresponding to the case in which the diatom begins rising with its concave side facing downwards. Finally, the equations were solved numerically using the RungeKutta method. The detailed derivation of the forces can be found in the Supplementary Material.

\section{Diatom submersion and motion simulations}

To more fully understand the underlying factors resulting in the change in orientation of the diatom frustules following $\mathrm{N}_{2}$ bubble agitation of the water's surface, simulations were conducted to analyze the interaction between the water surface agitation and the floating diatoms. To this end, two steps were undertaken to simulate the interactions among the $\mathrm{N}_{2}$ bubbles, water, and diatom frustules: the first step was to analyze the process of the diatoms' submersion into water, and the second step was to analyze the process of the diatom frustules floating back towards the water's surface following submersion. Of note, the simulations of the diatom's rise towards the water's surface address the same problem for which we sought solutions in the analytical method detailed above, thereby serving to verify both the experimental results and the analytical solutions, as well as facilitating a more comprehensive understanding of this phenomenon.

The process of a bubble rupturing was initially simulated using COMSOL (Supplementary Material, Supplementary Figures S3-S5). A cylindrical tank with a diameter of $32 \mathrm{~mm}$, a $10 \mathrm{~mm}$ depth of water, and a $10 \mathrm{~mm}$ layer of overlying $\mathrm{N}_{2}$ was employed for the simulation, which corresponded to our experimental setups. $\mathrm{N}_{2}$ bubbles ranging in size from 3.2 to $4.2 \mathrm{~mm}$ in diameter were initially simulated as originating $6 \mathrm{~mm}$ beneath the water's surface prior to release, and a time scale of $0.5 \mathrm{~s}$ was set to record the rupturing of the bubbles. The displacement of the water's surface induced by the rupture of the bubbles, was analyzed at a distance of $3 \mathrm{~mm}$ from the center of the rupturing bubble.

To verify the influence of the ruptures on the diatoms and analyze the diatoms' interaction with the water, a smoothed particle hydrodynamics (SPH) method was used ${ }^{35}$. In this method, to reduce the amount of calculation, a cross-section of the waterdiatom model (a 2D model) was simulated and analyzed. To simulate the effect of the rupturing bubble on the diatoms, a water tank of $3 \mathrm{~mm}$ in width and $0.8 \mathrm{~mm}$ in depth was simulated. A wave generator was simulated along one end of the tank to produce water surface agitation. Importantly, the water displacement data from the previous simulation were used as the motion data for the wave generator, which consequently mimics the water agitation following bubble rupture. A 2D model of a single C. sp. frustule was also built according to the aforementioned parameters. In the simulation, the frustule was placed $1 \mathrm{~mm}$ from the wave generator to avoid direct contact between these structures.

To simulate the interactions between the diatom frustule and the water following submersion, COMSOL models of a single diatom frustule being released from different depths beneath the water's surface $(100,200,300,400$, and $500 \mu \mathrm{m})$ were used. A frustule traveling through the water-air interface was also simulated. For both types of simulations, the same geometrical parameters and material properties of the frustules and water were used as in the analytical calculations.

In the frustule-release simulations, the COMSOL fluid-structure interaction (FSI) module was used. The governing equations for the incompressible fluid phase are the following Navier-Stokes equations of momentum and continuity:

$$
\begin{aligned}
& \rho\left(\frac{\partial \mathbf{u}}{\partial t}+\mathbf{u} \cdot \nabla \mathbf{u}\right)=\nabla \cdot\left[-p \mathbf{I}+\mu\left(\nabla \mathbf{u}+(\nabla \mathbf{u})^{\top}\right)\right]+\mathbf{f} \\
& \nabla \cdot(\rho \mathbf{u})=0
\end{aligned}
$$

where $\rho$ is the density of the fluid (in our case, water), $\mathbf{u}$ the velocity field of the fluid, $p$ the pressure, $\mu$ the dynamic viscosity, and $\mathbf{f}$ the external source term. Both the solid (frustule) and fluid (water) phases were subject to gravity.

In the interactions between the solid and fluid, the total forces exerted on the solid's surface are equal to the force on the fluid:

$$
\mathbf{F}_{T}=\mathbf{n} \cdot\left[-p \mathbf{I}+\mu\left(\nabla \mathbf{u}+(\nabla \mathbf{u})^{\top}\right)\right]
$$

Except for the boundary load exerted on the solid's surface, the fluid-structure boundary conditions of equal displacement and velocity were applied through a moving mesh. The frustule-water interactions were calculated under the influence of drag force, buoyancy force, and gravity.

To reduce the calculation load, a 2D model was constructed. In the FSI module, a deformable moving mesh was used for fluid domain calculations. In our application, the free travelling frustule structure may cause large deformations of the mesh, and therefore, automatic remeshing was also employed for solving the models. 


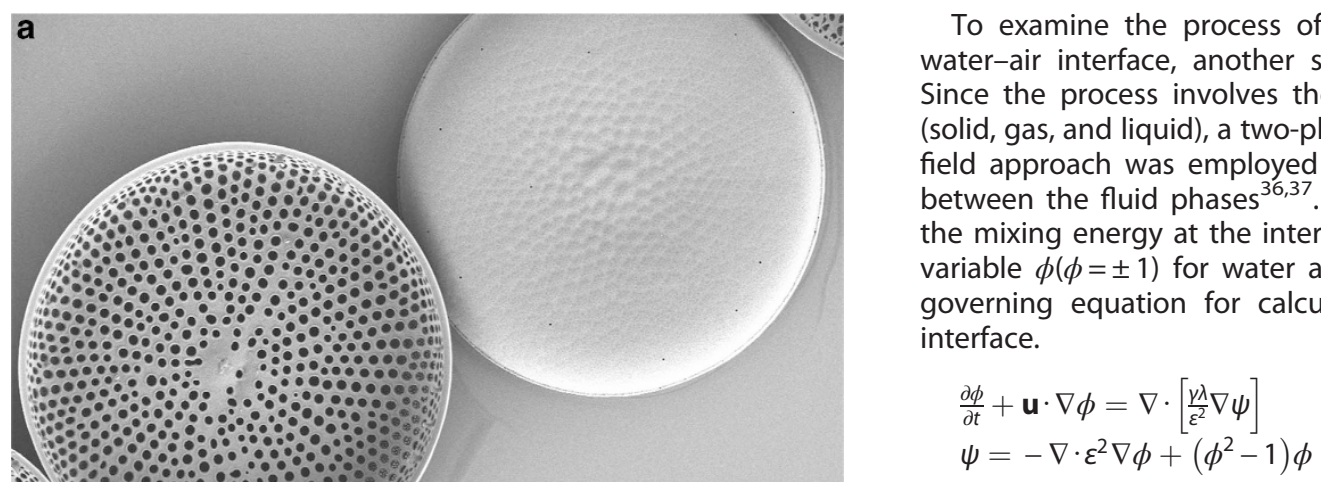

Here, $\gamma$ is the mobility, $\lambda$ is the magnitude of the mixing energy, and $\varepsilon$ is a capillary thickness ${ }^{36}$. Together with the aforementioned governing equations, boundary conditions, and moving mesh/ automatic remeshing conditions, the three-phase interaction problem was solved.

\section{RESULTS}

\section{Coscinodiscus species frustule morphology}

As noted above, the C. $s p$. diatom frustules shown in Figures $3 a$ and $b$ are dish-like, circular micron-scale valves, the diameter of which ranges from 50 to $150 \mu \mathrm{m}$. The total height of a C. sp. frustule is $\sim 12 \mu \mathrm{m}$. Along the concave surface of the frustules, micropores (termed foramen) with diameters $\sim 1 \mu \mathrm{m}$ are evenly distributed, while on the opposite, convex side, nanopores (termed cribellum) with diameters ranging in size to a minimum of $60 \mathrm{~nm}$ form the frustule surface. Between the foramen and cribellum pore layers, a layer featuring sub-micron pores (termed cribrum) is found in C. sp. diatom frustules (shown in Figure $3 c$ ) with diameters of $\sim 200 \mathrm{~nm}$. The microchambers enclosed by the foramen pore layer, the cribrum-cribellum layer, and the frustule side walls are termed areolae. The overall thickness of a single valve of the $C$. $s p$. diatom frustules consisting of the aforementioned hierarchical pore structure is approximately $1-1.2 \mu \mathrm{m}$.

\section{Uniformly oriented diatom monolayer formation}

Following surface modification with the release agent, the diatoms possessed a decreased surface energy, and their wetting became increasingly difficult. We hypothesize that this change allows the hierarchical pore and microchamber system to trap air within the frustule substructures, leading to the observed flotation of the frustules. After initial dispersion along the water's surface, compact diatom frustule monolayers can be formed with or without bubble agitation (Figures $3 \mathrm{~d}$ and e) before adding SDS solution to the water's surface. Note that upon addition of the SDS solution to the water's surface, the surface tension at the entry point of the SDS solution dropped instantaneously, while the tension of other areas of the surface remained static at that particular moment. This effect resulted in a contraction of the water's surface at higher tension, including the floating frustules, thereby yielding a compact monolayer in a short time. The area of the monolayer without bubble agitation ranged in size up to $1.1 \mathrm{~cm}^{2}$, nearly covering the entire surface of a cover slip, whereas the monolayer area after bubble-induced agitation decreased slightly, ranging in size up to $0.8 \mathrm{~cm}^{2}$. We found that by using the $\mathrm{N}_{2}$ bubble-induced agitation approach, up to nearly $90 \%$ of diatom frustules were found to orient with their concave side facing downwards (concave-down), although in the control group, $58.8 \pm 1 \%$ of the diatoms were adjusted to a concave-down orientation. It was hypothesized that the agitation from the rupturing of the $\mathrm{N}_{2}$ bubbles was the predominant factor affecting the orientation of the diatom frustules (concave-up versus concave-down) floating on the water's surface. Therefore, the 


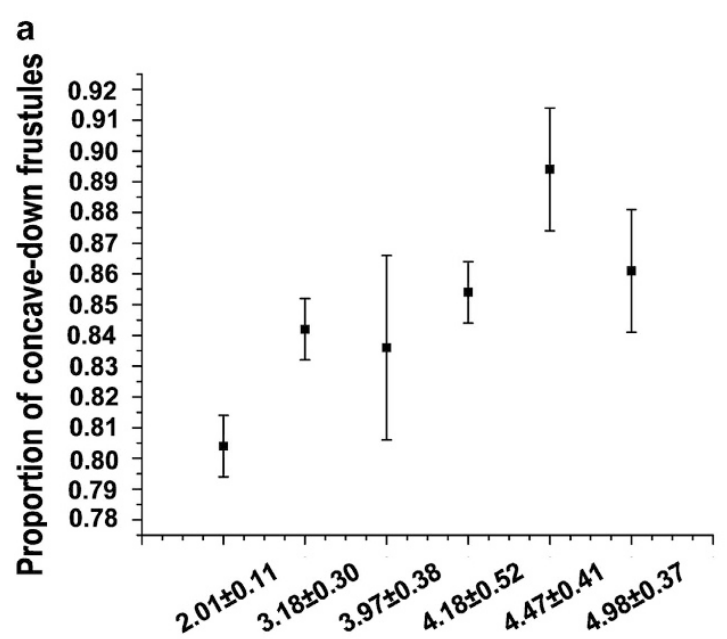

Bubble diameters (mm) b

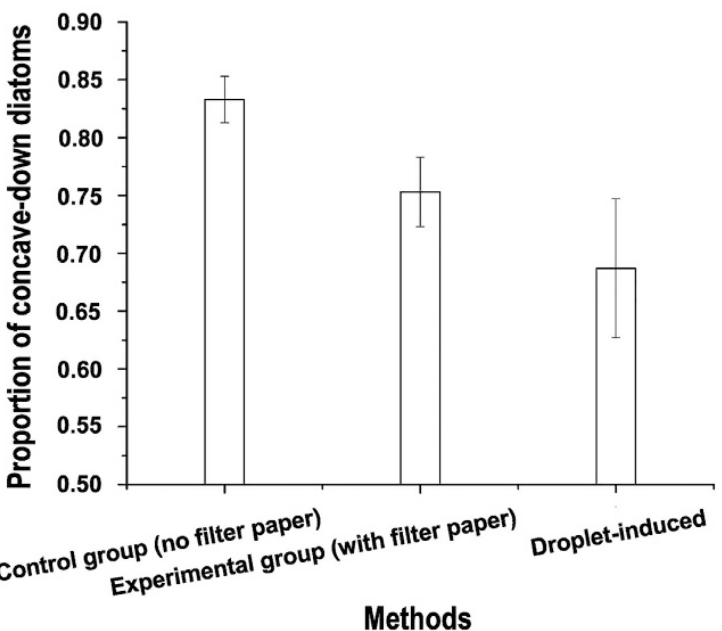

Figure 4 Proportion of concave-down diatoms as a function of different bubble sizes with the $\mathrm{N}_{2}$ bubbling method (a) and proportion of concave-down diatoms as a function of different agitation methods (b).

a

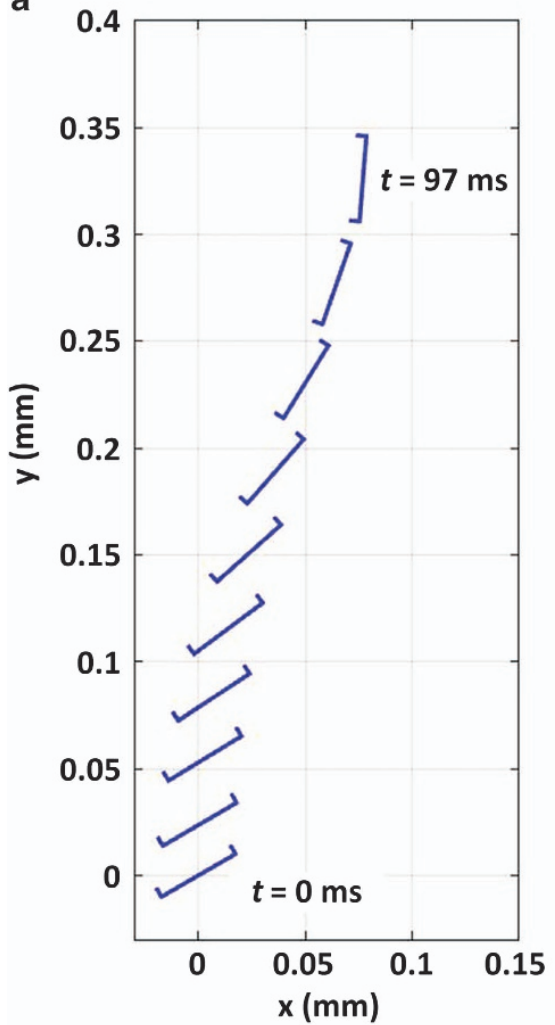

Rising trajectory of concave up diatom b

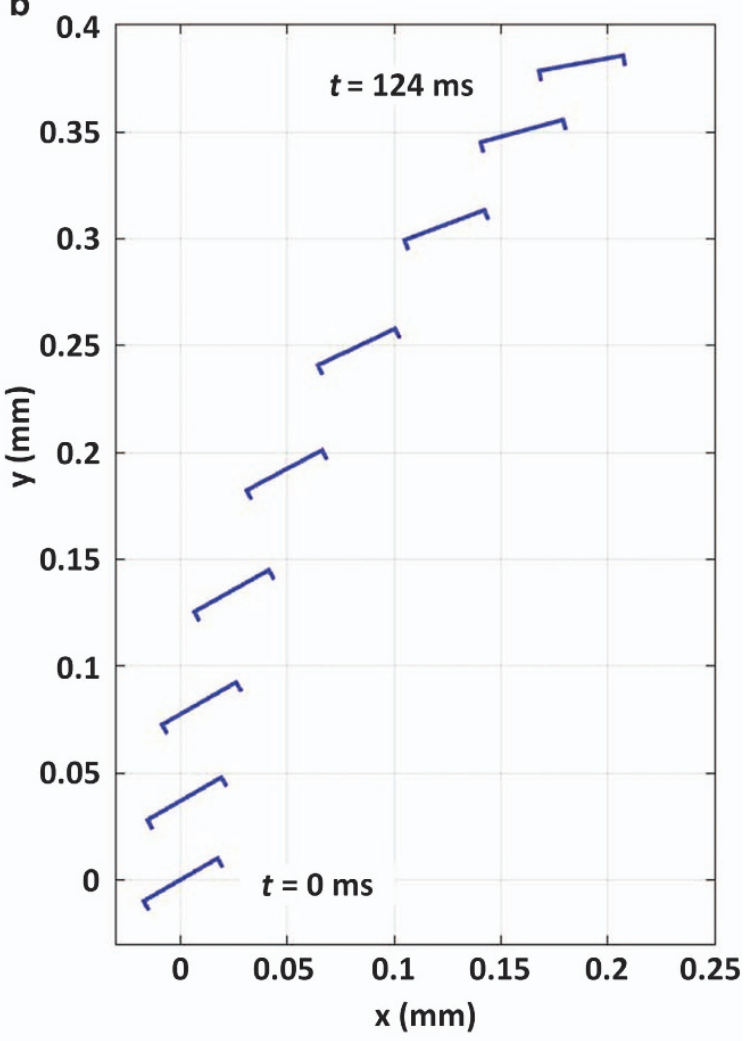

Rising trajectory of concave down diatom

Figure 5 Theoretical solutions of diatoms rising towards the water's surface following submersion, as a function of varying initial orientation. (a) A concave-up frustule's rising trajectory. (b) A concave-down frustule's rising trajectory. The initial tilt angle $(\theta(0))$ of both frustules is $30^{\circ}$.

correlation between the orientation of the diatoms and the agitation amplitude was analyzed using bubbles of varying size. The evaluation of the frustule orientation as a function of $\mathrm{N}_{2}$ bubble size is shown in Figure 4a, revealing that generally, as the bubble size increased, the concave-down diatom ratio increased.
By adding a layer of filter paper, we observed that during the bubble-induced agitation, the generated water jets from the bubbles wetted the paper. As the procedure continued, water jets were consecutively absorbed by the filter paper. In this manner, some of the water jets were prevented from falling back towards 
a
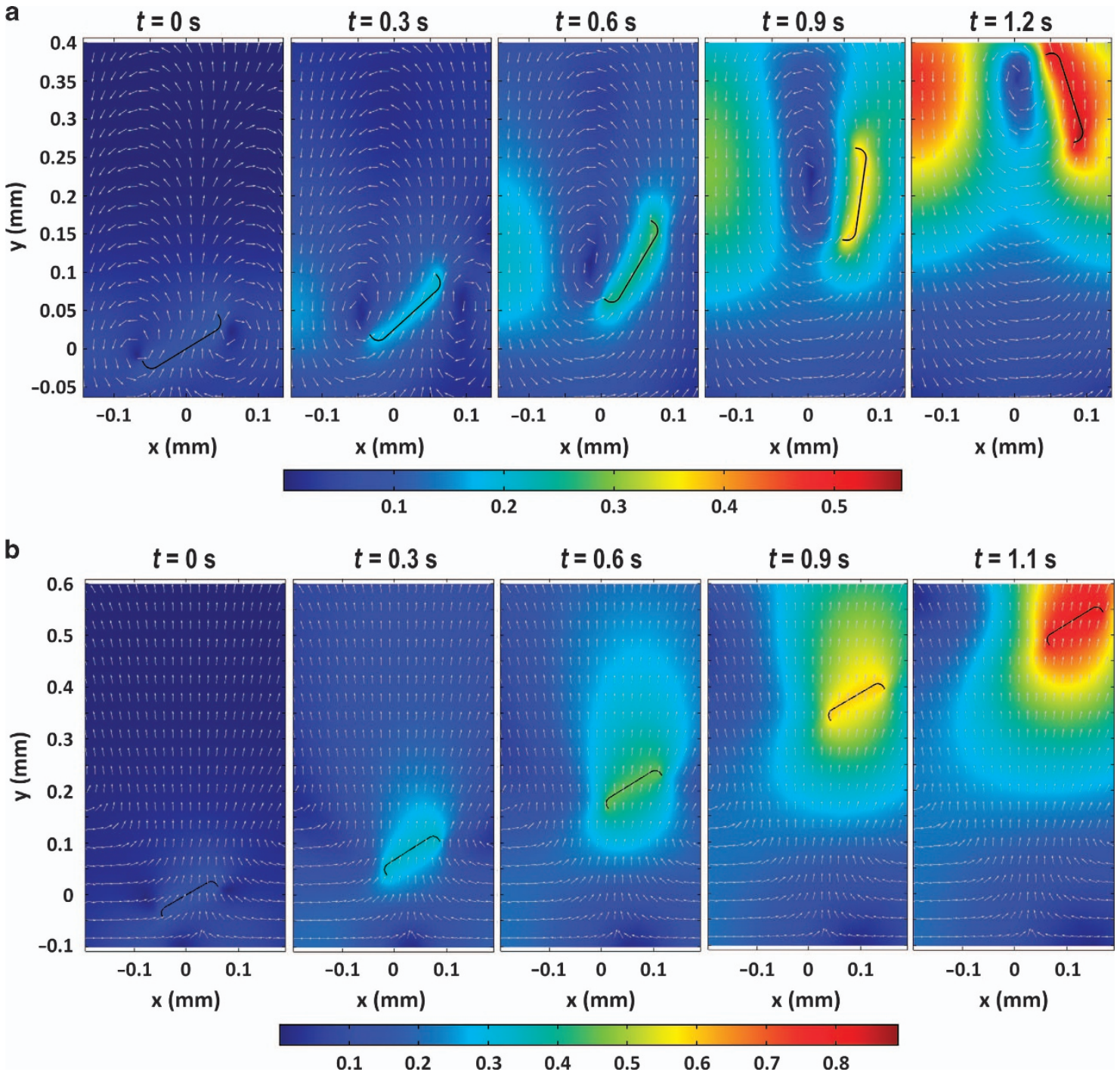

Figure 6 Simulated rising 2D diatom frustule and corresponding velocity field (unit $\mathrm{m} \mathrm{s}^{-1}$ ) of surrounding water. The initial tilt angle $(\theta(0))$ of both frustules was $30^{\circ}$. (a) The rising of a concave-up frustule. The frustule went through a self-adjusting process and eventually reoriented to a concave-down configuration. (b) The rising of a concave-down frustule. The tilt angle of the frustule oscillated between 29.9 and $32.6^{\circ}$ throughout the process.

the water's surface, mitigating their effect on the frustules along the water's surface. We found that $75.3 \pm 3 \%$ of the frustules were adjusted to a concave-down orientation in these experiments, although in the case of the control group without the addition of the filter paper, $83.3 \pm 2 \%$ of the frustules were adjusted to a concave-down orientation (Figure $4 \mathrm{~b}$ ). These results demonstrate that the water jets induced by the bubble rupture are likely to have a role in the final frustule orientation.

With the alternative method of diatom frustule agitation using the water droplet-based method, droplets were found to impact and submerge the majority of the floating diatoms. After the initial submersion, the majority of the diatoms would rise and again reach the water's surface. Following submersion of the diatoms and their subsequent rise towards the water's surface in this fashion, it was found that $68.7 \pm 6 \%$ of the diatoms were adjusted to a concave-down orientation.

\section{Analytical and simulation analyses of diatom orientation}

Using the analytical model we built and described above, the diatom frustule motion $(x(t), y(t)$ and $\theta(t))$ as a function of time may be solved for different initial angular deviations $(\theta(0))$. Figure 5 demonstrates the calculated movements of concave-up and concave-down diatom frustules. In both cases, $\theta(0)=30^{\circ}$ for demonstration purposes. It is evident from Figure $5 \mathrm{a}$ that through the adjustment of the drag force exerted on the diatoms, a concave-up diatom can be tilted to increase its angle with respect to the horizontal plane. The frustule's tilt angle, $\theta(t)$, will eventually reach $\pi / 2$ and beyond, flipping the frustule. Thus, through this motion, the initially concave-up frustule will change its orientation to a concave-down configuration. However, in the case of the initially concave-down configuration, the adjusting drag force does not induce an increase in the tilt angle (Figure 5b). Ultimately, these frustule motions will result in a concave-down orientation during the rise towards the water's surface following submersion.

To verify the experimental and analytical results, simulations were performed to study the frustules' behavior after $\mathrm{N}_{2}$ bubble rupture. It was found that the bubbles' rupturing generated waves with amplitudes ranging from 0.7 to $2.5 \mathrm{~mm}$ in size (Supplementary Material, Supplementary Figure S3), whereas the sizes of the frustules ranged from 50 to $150 \mu \mathrm{m}$. Given waves of this size, some of the diatom frustules were readily submerged 


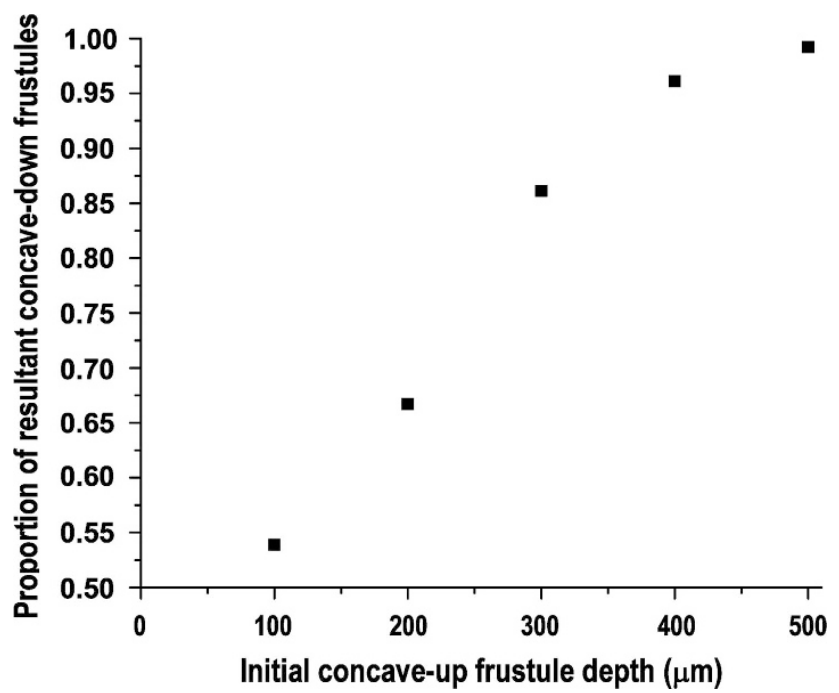

Figure 7 Simulation results of the influence of the initial depth of a concave-up diatom frustule on the final concave-down ratio.

beneath the water's surface (Supplementary Video). When submersion was not achieved, the frustule was found to be moved violently and randomly along the water's surface.

The COMSOL simulations showed a similar behavior to the analytical approach. Once submerged, the frustule initially travels with the underlying water currents and eventually floats back towards the water's surface, self-adjusting to a concave-down orientation during this process (Figure 6).

Assuming an equal chance of any initial tilt angle $(\theta(0))$ after being submerged, we can calculate the corresponding proportion of diatoms that could eventually reach the critical angle of $\pi / 2$. In the COMSOL simulations, an initial depth of $100 \mu \mathrm{m}$ was first analyzed with varying initial tilt angles $(\theta(0))$. When $\theta(0)$ was $\geqslant 83^{\circ}$ (corresponding to $53.9 \%$ of all submerged diatoms), the diatoms' $\theta$ became $\pi / 2$ as they rose to the water's surface. When the depth was increased to $200 \mu \mathrm{m}, 66.7 \%$ were adjusted to $\pi / 2$. Furthermore, when the initial depth was 300, 400, and $500 \mu \mathrm{m}, 86.1,96.1$ and $99.2 \%$ of all submerged diatoms, respectively, were adjusted to $\pi / 2$, indicating that, as the submersion depths of the diatoms increased, increasing numbers of diatom frustules would orient towards a concave-down configuration (Figure 7).

For the solid-liquid-gas interactions, we are mainly interested in determining whether the frustules that have already reached $\pi / 2$ will eventually breach the water-air interface and continue to roll over to assume a concave-down configuration. We are also interested in whether frustules with a concave-down or concaveup configuration will maintain their posture as they breach the water-air interface. As illustrated in Figure 8, both the frustule with $\theta(0)=90^{\circ}$ and the concave-down frustule with $\theta(0)=45^{\circ}$ finally breached the water-air interface as they reoriented themselves to a concave-down configuration. However, the concave-up frustule with $\theta(0)=45^{\circ}$ arrived at the surface with a concave-up configuration. The simulated phenomenon indicated that as diatoms travel through the water's surface, the interaction among the frustule, water and air will not suddenly change the turning direction of the frustules.

\section{DISCUSSION}

This study reports that through the process of $\mathrm{N}_{2}$ bubble-induced agitation, diatom frustules achieved a highly uniform concavedown orientation. Subsequently, we sought to examine and more completely understand this phenomenon through experimental and analytical inquiry, along with simulations.

We first conducted additional experiments using filter paper to mitigate the effects of bubble rupture-induced water jets interacting with the diatom frustules. We hypothesize that the decrease in concave-down frustule ratio achieved in this fashion is due to a less efficient submersion of diatom frustules, with submersion being fundamental to the capacity of the frustule to reorient in the concave-down orientation. In addition to the analysis of the bubble agitation method, a water droplet-induced agitation method was also employed, and the results also corresponded well with the subsequent analytical and simulation results-nearly $70 \%$ of the diatoms were reoriented to a concavedown configuration. In addition, in the COMSOL simulations of rupturing bubbles, water jets (Supplementary Materials Supplementary Figure S4) and sinking currents (Supplementary Materials Supplementary Figure S5) were observed near the bubbles after they burst. Prior studies ${ }^{38}$ have also discussed the possible influence of such rupturing upon floating objects, reporting that when the radius of the bubble is greater than a floating 'ship' and when the floating 'ship' is positioned close to the outer rim of the bubble when it reaches the water's surface, it is possible for the bubble to cause the 'ship' to sink, lending further support to our conclusion that submersion is a major contributing process in adjusting the diatoms' orientation. We thus conclude that the predominant effect of the bubble rupture is the creation of surface agitation, leading to submersion of the diatom frustules, and that this submersion is fundamental to the effect on the diatom uniformity observed using $N_{2}$ bubbleinduced water surface agitation.

When comparing the water droplet-induced agitation with the bubble-induced agitation, however, fewer water droplets than bubbles were generated during a given period of time. In addition, since the water droplets were generated individually and manually, the surface area of the agitation was limited at any given time, although the continuous motion of the bubbleinduced agitation affected the entire surface of the water continuously. We hypothesize that these differences between the methods of water surface agitation likely resulted in the observed smaller flipped-diatom ratio of the water droplet method compared with the bubble method.

To summarize, based on the experimental results, we conclude that after bubble rupture, diatoms are submerged beneath the water's surface and undergo a series of interactions with water. Water drag alters the orientation of the submerged diatoms, and due to the imbalance in forces exerted on the frustule, a concaveup frustule rising towards the water's surface is found to eventually reorient into a concave-down configuration. However, as a concave-down frustule rises in the water following submersion, no reorienting occurs, eventually resulting in the frustule floating on the water's surface in a concave-down state.

In this paper, the analytical model serves as a qualitative description of the frustule's rising in water, given the requisite simplifications employed for the ease of calculations. The discrepancy between the analytical and simulation results can be attributed to the different methods employed to analyze the model. In the analytical approach, we employed momentum change and added mass to account for drag forces and viscous forces exerted on the diatom frustule body, both of which reflect simplifications of the fluid-structure interaction. However, in the simulation model, pressure distribution and viscous stress were evaluated, yielding more accurate predictions of the fluidstructure interaction.

The numerical method is applied for qualitative analysis, noting that it represents a simplified model; the dish-like 3D structure and the micro/nanopores of the diatom frustules are not considered. Nevertheless, the analytical solutions and simulations provide a degree of insight into the likely behavior of the 

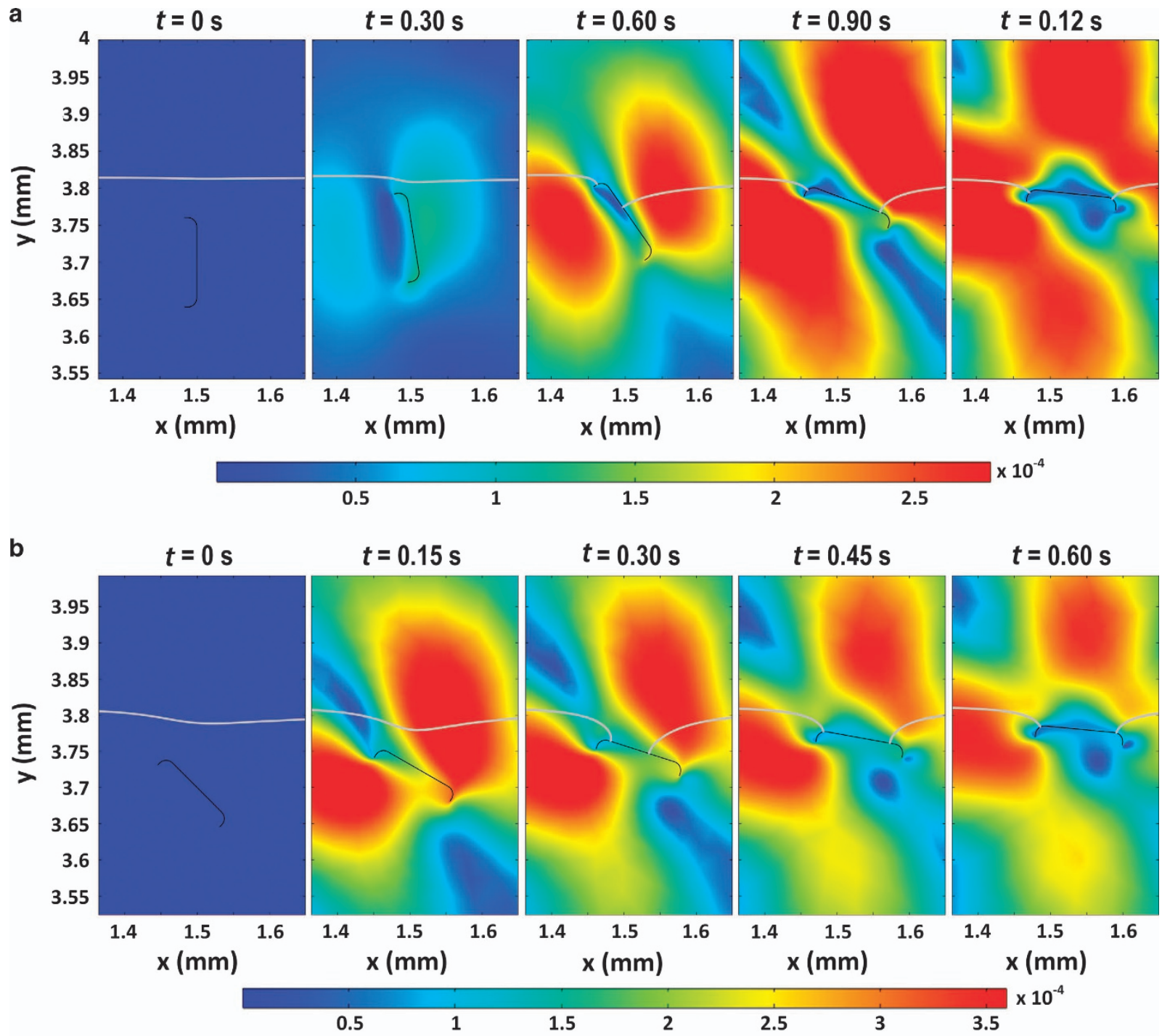

C

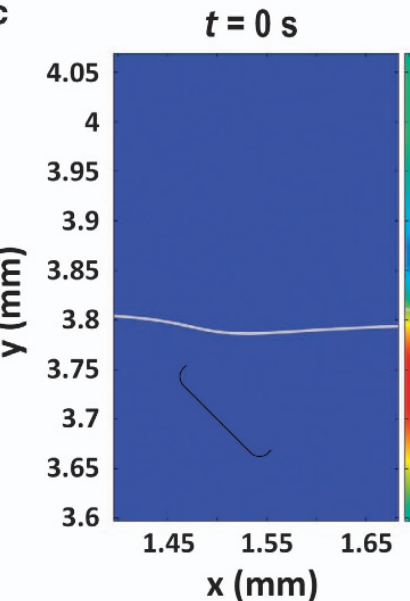

0.5

1

1.5

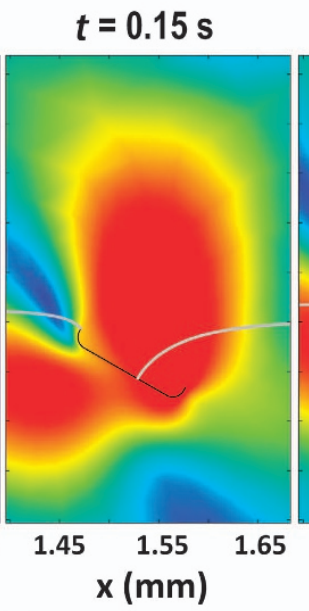

$$
t=0.45 \mathrm{~s}
$$

$t=0.60 \mathrm{~s}$
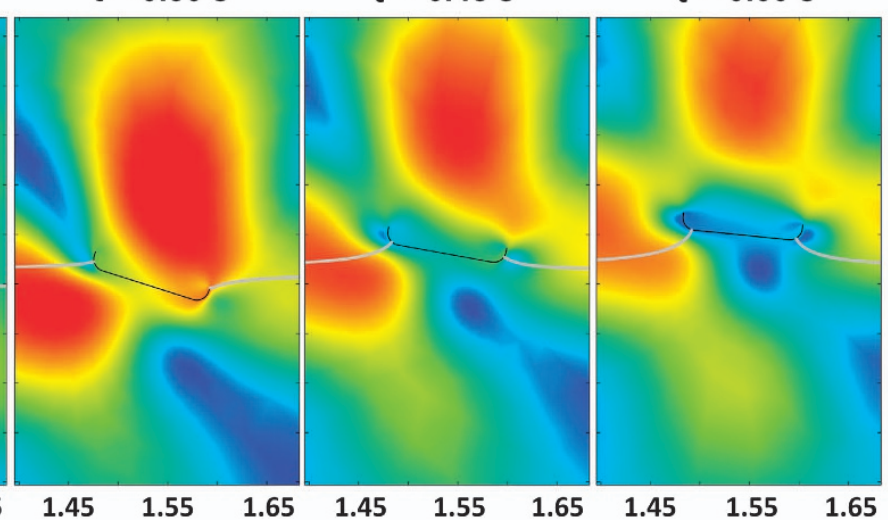

0.5

1

1.5

2

2.5

3

3

Figure 8 Frustules breaching the water-air interface and corresponding velocity field (unit $\mathrm{m} \mathrm{s}^{-1}$ ). Gray curves indicate the water-air interface. All frustules were released with 0 velocity. (a) The initial tilt angle $(\theta(0))$ of the frustule was $90^{\circ}$. (b) The rising of a concave-down frustule with an initial angle of $45^{\circ}$. In (a) and (b), both frustules passed through the interface and eventually reoriented to a concave-down configuration. (c) The rising of a concave-up frustule with an initial angle of $45^{\circ}$, which ultimately ended on the water's surface with a concave-up configuration. 
submerged frustules. They indicate that, given a sufficient rising distance (following a sufficient submersion depth), all diatoms may reorient towards a concave-down configuration on the water's surface following submersion. Given the combined insights derived from the analytical and simulation results, we conclude that the interaction between rising diatoms and water is a significant factor in determining the ultimate orientation of the diatoms.

The analytical and simulation results yield useful initial insight into the frustule agitation/submersion process and address the experimentally observed uniform orientation phenomenon of the C. sp. frustules. However, the nature of the agitations, due to the rapid and consecutive rupturing of bubbles, means that the direct recording and analysis of the diatoms' submersion and subsequent motion during their rise towards the surface is highly technically challenging. Additional experimental improvements such as directly releasing modified diatoms in water at precisely controlled submersion depths will allow us to more closely scrutinize the validity of the analytical and simulation results with respect to the experimental findings. Furthermore, high-speed imaging techniques have the potential for direct investigation of the interaction between the diatom frustules and the rupturing bubbles.

\section{CONCLUSION}

In this paper, an experimentally observed phenomenon of C. $s p$. frustules orienting preferentially in a specific, concave-down manner following agitation by $\mathrm{N}_{2}$ bubbles is reported. It was found that following bubble agitation, up to nearly $90 \%$ of the frustules were arranged into monolayers with their concave sides facing downward. Furthermore, it was revealed that with an increase in the bubble size employed during bubble-induced agitation, the concave-down diatom ratio was increased. It was concluded from both analytical study and simulations that the concave-down orientation was ultimately favored following the diatoms' rise towards the water's surface after submersion. The method in this paper has the potential to evolve into a relatively convenient fabrication procedure for batch arranging the frustules in a uniform orientation. The capacity to manipulate diatoms in such a manner could be employed in furthering a variety of biologically enabled technologies, from sensor fabrication to light-trapping energy applications. Beyond the specific case of diatom frustule orientation examined herein, the convenient agitation method employed to adjust a number of asymmetrically shaped micro particles on the water's surface, along with the corresponding analytical and simulation models, may be generalized and could potentially be beneficial for the manipulation of myriad asymmetrically shaped micro devices/ particles.

\section{ACKNOWLEDGEMENTS}

This research is in part supported by the National Science Foundation (NSF ECCS-1202304). We thank the Boston University Nanotechnology Innovation Center and the Photonics Center at Boston University for funding and technical support.

\section{COMPETING INTERESTS}

The authors declare no conflict of interest.

\section{REFERENCES}

1 Gordon R, Aguda BD. Diatom morphogenesis: Natural fractal fabrication of a complex microstructure. Proceedings of the Annual International Conference of the IEEE Engineering in Medicine and Biology Society, Part 1/4: Cardiology and Imaging; 4-7 Nov 1988; New Orleans, LA, USA; 1988: 4-7.

2 Parkinson J, Gordon R. Beyond micromachining: The potential of diatoms. Trends in Biotechnology 1999; 17: 190-196.
3 De Stefano M, De Stefano L, Congestri R. Functional morphology of micro-and nanostructures in two distinct diatom frustules. Superlattices and Microstructures 2009; 46: 64-68.

$4 \mathrm{Hamm}$ CE, Merkel R, Springer $\mathrm{O}$ et al. Architecture and material properties of Diatom shells provide effective mechanical protection. Nature 2003; 421: 841-843.

5 Gordon R, Losic D, Tiffany MA et al. The glass menagerie: Diatoms for novel applications in nanotechnology. Trends in Biotechnology 2009; 27: 116-127.

6 Kieu K, Li C, Fang Y et al. Structure-based optical filtering by the silica microshell of the centric marine diatom Coscinodiscus wailesii. Optics Express 2014; 22: 15992-15999.

7 Romann J, Valmalette J-C, Røyset A et al. Optical properties of single diatom frustules revealed by confocal microspectroscopy. Optics Letters 2015; 40: 740-743.

8 Losic D, Rosengarten G, Mitchell JG et al. Pore architecture of diatom frustules: Potential nanostructured membranes for molecular and particle separations. Journal of Nanoscience and Nanotechnology 2006; 6: 982-989.

9 Dolatabadi JEN, de la Guardia M. Applications of diatoms and silica nanotechnology in biosensing, drug and gene delivery, and formation of complex metal nanostructures. Trends in Analytical Chemistry 2011; 30: 1538-1548.

10 Townley HE, Parker AR, White-Cooper H. Exploitation of diatom frustules for nanotechnology: Tethering active biomolecules. Advanced Functional Materials 2008; 18: 369-374.

11 Lin K-C, Kunduru V, Bothara $M$ et al. Biogenic nanoporous silica-based sensor for enhanced electrochemical detection of cardiovascular biomarkers proteins. Biosensors and Bioelectronics 2010; 25: 2336-2342.

12 Bao Z, Weatherspoon MR, Shian S et al. Chemical reduction of three-dimensional silica micro-assemblies into microporous silicon replicas. Nature 2007; 446: $172-175$.

13 De Stefano L, Rendina I, De Stefano M et al. Marine diatoms as optical chemical sensors. Applied Physics Letters 2005; 87: 233902.

14 Gale DK, Gutu T, Jiao J et al. Photoluminescence detection of biomolecules by antibody-functionalized diatom biosilica. Advanced Functional Materials 2009; 19: 926-933.

15 De Tommasi E, De Luca A, Lavanga L et al. Biologically enabled sub-diffractive focusing. Optics Express 2014; 22: 27214-27227.

16 Hsu S-H, Paoletti C, Torres M et al. Light transmission of the marine diatom Coscinodiscus wailesii. Proceedings of SPIE Smart Structures and Materials and Nondestructive Evaluation and Health Monitoring 2012; 8339: 83390F-83398F.

17 Toster J, lyer KS, Xiang W et al. Diatom frustules as light traps enhance DSSC efficiency. Nanoscale 2013; 5: 873-876.

18 Chen X, Wang C, Baker E et al. Numerical and experimental investigation of light trapping effect of nanostructured diatom frustules. Scientific Reports 2015; 5: 11977.

19 Li A, Cai J, Pan J et al. Multi-layer hierarchical array fabricated with diatom frustules for highly sensitive bio-detection applications. Journal of Micromechanics and Microengineering 2014; 24: 025014.

20 Pan J, Cai J, Zhang D et al. Micro-arraying of nanostructured diatom microshells on glass substrate using ethylene-vinyl acetate copolymer and photolithography technology for fluorescence spectroscopy application. Physica E: Low-Dimensional Systems and Nanostructures 2012; 44: 1585-1591.

$21 \mathrm{Im} \mathrm{SH}$, Lim YT, Suh DJ et al. Three-dimensional self-assembly of colloids at a water-air interface: A novel technique for the fabrication of photonic bandgap crystals. Advanced Materials 2002; 14: 1367-1369.

22 Quan Z, Fang J. Superlattices with non-spherical building blocks. Nano Today 2010; 5: 390-411.

23 Bowden N, Terfort A, Carbeck J et al. Self-assembly of mesoscale objects into ordered two-dimensional arrays. Science 1997; 276: 233-235.

24 Liu Y, Wang S, Lee JW et al. A floating self-assembly route to colloidal crystal templates for 3D cell scaffolds. Chemistry of Materials 2005; 17: 4918-4924.

25 Denkov N, Velev O, Kralchevski $\mathrm{P}$ et al. Mechanism of formation of two-dimensional crystals from latex particles on substrates. Langmuir 1992; 8: 3183-3190.

26 Nagayama K. Two-dimensional self-assembly of colloids in thin liquid films. Colloids and Surfaces A: Physicochemical and Engineering Aspects 1996; 109: 363-374.

27 Kralchevsky PA, Nagayama K. Capillary interactions between particles bound to interfaces, liquid films and biomembranes. Advances in Colloid and Interface Science 2000; 85: 145-192.

28 Kralchevsky PA, Denkov ND. Capillary forces and structuring in layers of colloid particles. Current Opinion in Colloid \& Interface Science 2001; 6: 383-401.

29 Wang Y, Pan J, Cai J et al. Floating assembly of diatom Coscinodiscus sp. microshells. Biochemical and Biophysical Research Communications 2012; 420: 1-5.

30 Kwon SY, Park S, Nichols WT. Self-assembled diatom substrates with plasmonic functionality. Journal of the Korean Physical Society 2014; 64: 1179-1184. 
31 Zhang D, Wang Y, Pan J et al. Separation of diatom valves and girdle bands from Coscinodiscus diatomite by settling method. Journal of Materials Science 2010; 45: 5736-5741.

32 Smol JP, Stoermer EF. The diatoms: Applications for the environmental and earth sciences. Cambridge University Press: Cambridge, UK, 2010.

33 Newman JN. Marine hydrodynamics. MIT Press: Cambridge, MA, USA, 1977.

34 Veritas N. Environmental conditions and environmental loads. Det Norske Veritas: Høvik, Norway, 2000.

35 Crespo AJC, Domínguez JM, Rogers BD et al. DualSPHysics: Open-source parallel CFD solver based on smoothed particle hydrodynamics (SPH). Computer Physics Communications 2015; 187: 204-216.

36 Yue $\mathrm{P}$, Feng J, Liu C et al. A diffuse-interface method for simulating two-phase flows of complex fluids. Journal of Fluid Mechanics 2004; 515: 293-317.

$37 \mathrm{Kim} \mathrm{J.} \mathrm{Phase-field} \mathrm{models} \mathrm{for} \mathrm{multi-component} \mathrm{fluid} \mathrm{flows.} \mathrm{Communications} \mathrm{in}$ Computational Physics 2012; 12: 613-661.
38 May DA, Monaghan JJ. Can a single bubble sink a ship? American Journal of Physics 2003; 71: 842-849.

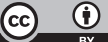

This work is licensed under a Creative Commons Attribution 4.0 International License. The images or other third party material in this article are included in the article's Creative Commons license, unless indicated otherwise in the credit line; if the material is not included under the Creative Commons license, users will need to obtain permission from the license holder to reproduce the material. To view a copy of this license, visit http://creativecommons.org/licenses/ by/4.0/

(c) The Author(s) 2016

Supplementary Information for this article can be found on the Microsystems \& Nanoengineering website (http://www.nature.com/ micronano) 